\title{
Physical Stress from Colonoscopy and Polypectomy in Elderly Individuals: Evaluation Using Damage Biomarkers in Blood
}

\author{
Hiroaki Igarashi, Hiroko Yamashita, Kiyoshi Tsuchiya, Yuki Hanaoka, Dai Sugimoto, Itsuro Ogata \\ Department of Gastroenterology, Kawakita General Hospital, Tokyo, Japan
}

Email address:

igarashi@kawakita.or.jp (H. Igarashi)

\section{To cite this article:}

Hiroaki Igarashi, Hiroko Yamashita, Kiyoshi Tsuchiya, Yuki Hanaoka, Dai Sugimoto, Itsuro Ogata. Physical Stress from Colonoscopy and Polypectomy in Elderly Individuals: Evaluation Using Damage Biomarkers in Blood. American Journal of Clinical and Experimental Medicine. Vol. 6, No. 2, 2018, pp. 33-39. doi: 10.11648/j.ajcem.20180602.11

Received: February 3, 2018; Accepted: February 25, 2018; Published: March 21, 2018

\begin{abstract}
Colonoscopy is routinely performed worldwide, but it is still a physically stressful screening method for colorectal cancer, particularly in elderly individuals. No previous study has evaluated physical stress from colonoscopy itself and polypectomy using blood-derived biomarkers. This study aimed to evaluate physical stress from colonoscopy and polypectomy in elderly individuals using white blood cell counts (WBCs) and C-reactive protein (CRP) levels, which are widely used blood-based biomarkers that respond quickly to tissue damage. In this single-center, retrospective observational study, 109 consecutive patients, aged $\geq 80$ years, were enrolled. In all patients, WBCs and CRP levels were measured before and half-a-day after colonoscopy. The changes were subsequently compared. Patients were divided into two groups: those with polypectomy $(\mathrm{P}+)$ and those without polypectomy (P-). The changes in WBCs and CRP levels were determined and compared between the two groups. In the $\mathrm{P}+$ group, correlations between the number of resected polyps and changes in WBCs and CRP levels were also evaluated. Of the 109 patients, there were 48 men and 61 women with a mean age of $83.9 \pm 3.1$ years (range, $80-91$ years); 58 patients received polypectomy. In all patients, the mean WBC after colonoscopy showed no significant change from that before colonoscopy (before: $5400 / \mu \mathrm{L}$, after: $5200 / \mu \mathrm{L}, P=0.092$ ), and the mean CRP level showed a slight elevation after colonoscopy (before: $0.05 \mathrm{mg} / \mathrm{dL}$, after: $0.08 \mathrm{mg} / \mathrm{dL}, P<0.001$ ). Compared to before colonoscopy, the mean WBC after colonoscopy decreased slightly in the $\mathrm{P}$ - group (before $5400 / \mu \mathrm{L}$, after $4900 / \mu \mathrm{L}, P=0.002$ ), but showed no significant difference in the $\mathrm{P}+$ group $(5400 / \mu \mathrm{L}, 5400 / \mu \mathrm{L}, P=0.48)$. Conversely, the mean CRP level increased very slightly in both the $\mathrm{P}$ - and $\mathrm{P}+$ groups (from $0.06 \mathrm{mg} / \mathrm{dL}$ to $0.07 \mathrm{mg} / \mathrm{dL}, P=0.003$; from $0.04 \mathrm{mg} / \mathrm{dL}$ to $0.08 \mathrm{mg} / \mathrm{dL}, P<0.001$, respectively), but it was not significantly different between the groups. The elevation of both WBCs and CRP levels showed only minor correlations with the number of resected polyps. In conclusion, colonoscopy can be well tolerated even by elderly individuals because physical stress from colonoscopy, with respect to WBCs and CRP levels, was minimal even if polypectomy was performed.
\end{abstract}

Keywords: Colonoscopy, Elderly People, Physical Stress, C-Reactive Protein (CRP), White Blood Cell Count (WBC)

\section{Introduction}

Colorectal cancer is one of the most common cancers in the world. It is the third leading cause of cancer-related deaths in men and the leading cause in women in Japan [1]. Since 1987, annual population-based colorectal cancer screening using an established and recommended screening method, i.e., an immunochemical fecal occult blood test [2-8], has been successfully performed in Japan. Examinees collect fecal samples for two consecutive days, and if the sample is positive for occult blood, they are referred for colonoscopy, which is a more detailed examination. Colonoscopy is essential for detecting colorectal cancer [7-9] and has become a common examination in patients regardless of age. As there is no upper age limit for colorectal cancer screening in Japan, gastroenterologists accept many elderly individuals for colonoscopy.

In general, any medical screening method that targets healthy people must be minimally invasive and mostly tolerable for patients who are usually asymptomatic. However, 
both diagnostic colonoscopy and polypectomy, which is often performed during colonoscopy and considered a type of surgery, may be considered as invasive, as they are both physically and psychologically stressful, especially in vulnerable elderly individuals.

It is well known that complications from colonoscopy, including both major and minor, are more frequent than those arising from any other screening method for colorectal cancer $[8,10]$, and colonoscopy can affect not only the intestinal tract, but also the cardiopulmonary system during the procedure, especially in patients who have comorbid diseases. Major complications from colonoscopy, such as perforation and hemorrhage, have been noted in a large number of studies, but minor complications, including physical and psychological stresses, have not received attention. It is important in clinical settings to understand how elderly individuals are affected by colonoscopy physically and psychologically; in other words, a sense of how stressful it is to undergo colonoscopy.

A few studies have evaluated physical stress caused by colonoscopy using salivary alpha amylase activity, which responds very quickly to stress $[11,12]$, but no previous studies have evaluated physical stress caused by colonoscopy using blood-derived data. Furthermore, no study has evaluated physical stress caused by polypectomy.

The aim of this study was to evaluate physical stress caused by colonoscopy and accompanying polypectomy in elderly individuals by using white blood cell counts (WBCs) and C-reactive protein (CRP) levels, which are well-known and common blood-based biomarkers that respond quickly to any damage or inflammation in the body.

\section{Patients and Methods}

This retrospective observational study was performed at a single center. One hundred nine consecutive in-hospital patients who underwent colonoscopy with or without polypectomy at our hospital between 2008 and 2012 were enrolled. Patients were hospitalized to undergo colonoscopy due to positive results of the fecal occult blood test for colorectal cancer screening. Only patients aged $\geq 80$ years were enrolled in this study because patients aged $\geq 80$ years were recommended to be hospitalized for three days to prepare for and undergo colonoscopy safely. Consequently, the collection of sufficient blood data for this study was performed during the patients' stay. Written informed consent was obtained from each patient on admission.

Prior to colonoscopy, for bowel preparation, patients consumed two liters of a liquid medicine, including polyethylene glycol-electrolyte solution, on the morning of the examination day. All colonoscopies were performed by specialists in the field of endoscopy. The colonoscopy devices used were the CF-Q260AI or PCF-Q260AI (Olympus, Tokyo, Japan), and selection of the device was at the examiner's discretion. Conscious sedation was administered to 25 patients $(23 \%)$ according to the examiner's preference: $35 \mathrm{mg}$ of pethidine in 22 patients $(20 \%), 2.5 \mathrm{mg}$ of midazolam in two patients $(2 \%)$, and $15 \mathrm{mg}$ of pentazocine in one patient $(1 \%)$. To reduce bowel movement, 5 or $10 \mathrm{mg}$ of butyl scopolamine (52 patients [48\%]) or $1 \mathrm{mg}$ of glucagon (28 patients [26\%]) was intravenously administered before the procedure. We did not use carbon dioxide $\left(\mathrm{CO}_{2}\right)$ insufflation. Polypectomy using electrocautery was performed when adenomatous or cancerous polyps were found, except when the patient was taking antithrombotic compounds. In polypectomy cases, the choice of either snaring polypectomy or hot forceps polypectomy was made on the spot by the examiner mainly based on the polyp size. WBCs and CRP levels were both measured on the day before and one-half day after colonoscopy in each patient. Changes in WBCs and CRP levels before and after colonoscopy were determined. Patients were categorized into two subgroups, namely those with polypectomy $(\mathrm{P}+)$ and those without polypectomy (P-). The changes in WBCs and CRP levels were determined and compared between the two groups. In the $\mathrm{P}+$ group, the correlation between the number of resected polyps and changes in WBCs and CRP levels were also evaluated. The WBC was determined by flow cytometry (Cell-Dyn Sapphire, Abbott Diagnostic, Santa Clara, CA, USA), and CRP level was measured by latex flocculation turbidimetry (CRP-Latex $\times 2$, Denka-Seiken, Tokyo, Japan).

Patients who developed major complications, including massive bleeding and colonic perforation, by the next day or those who had advanced colorectal cancer were excluded from this study because their blood data may have been affected by the adverse event or cancer itself. Patients who received biopsy (not polypectomy) were also excluded because obtaining a biopsy can cause minor tissue damage to the colon, which could affect the results. Patients who showed elevated WBCs $(>8500 / \mu \mathrm{L}$, beyond the normal upper limit at our hospital) or elevated CRP levels $(>0.3 \mathrm{mg} / \mathrm{dL}$, beyond the normal upper limit at our hospital) before colonoscopy were also excluded because they may have already considerable stress or inflammatory disease.

The Wilcoxon signed rank test or the Mann-Whitney test was used for evaluating changes in WBCs and CRP levels, and the Spearman rank correlation coefficient was used for evaluating the correlations between the number of resected polyps and changes in WBCs and CRP levels. Statistical significance was set at $P<0.05$.

This study was approved by the ethics committee of our hospital.

\section{Results}

Of the 109 patients enrolled in this study, there were 48 men and 61 women with a mean age of $83.9 \pm 3.1$ years (range, $80-91$ years). Among these, 58 patients received polypectomy $(\mathrm{P}+$ group) and 51 did not ( $\mathrm{P}-$ group) (Table 1$)$.

Table 1. Characteristics of the study patients $(n=109)$.

\begin{tabular}{ll}
\hline Age $($ years $)$ & $83.9 \pm 3.1$ \\
Male $(\%)$ & $48(44.0)$ \\
No. of polypectomies $(0 / 1 / 2 / 3 / \geq 4)$ & $51 / 31 / 11 / 10 / 6$ \\
WBC before colonoscopy $(/ \mu \mathrm{L})$ & $5,400[4,600-6,000]$ \\
CRP before colonoscopy $(\mathrm{mg} / \mathrm{dL})$ & $0.05[0.03-0.09]$ \\
\hline
\end{tabular}


In all patients, the mean WBC after colonoscopy showed no significant change compared with that before colonoscopy (before: $5400 / \mu \mathrm{L}$, after: $5200 / \mu \mathrm{L}, P=0.092$ ), and mean CRP

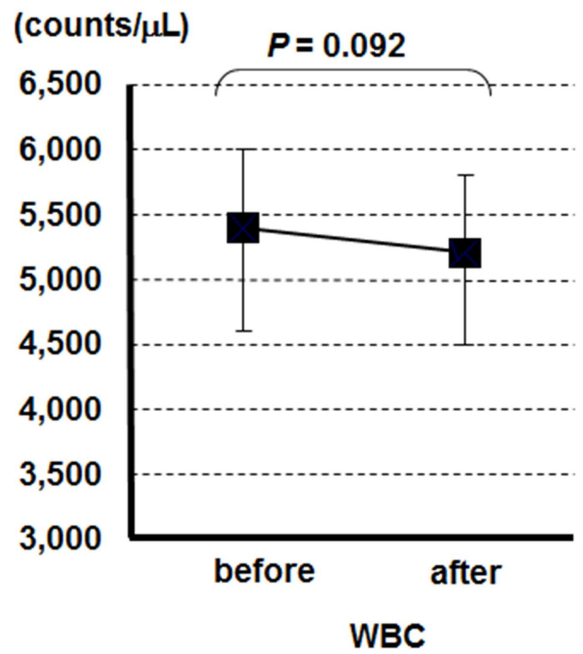

level showed a slight elevation after colonoscopy (before: $0.05 \mathrm{mg} / \mathrm{dL}$, after: $0.08 \mathrm{mg} / \mathrm{dL}, P<0.001$ ) (Figure 1).

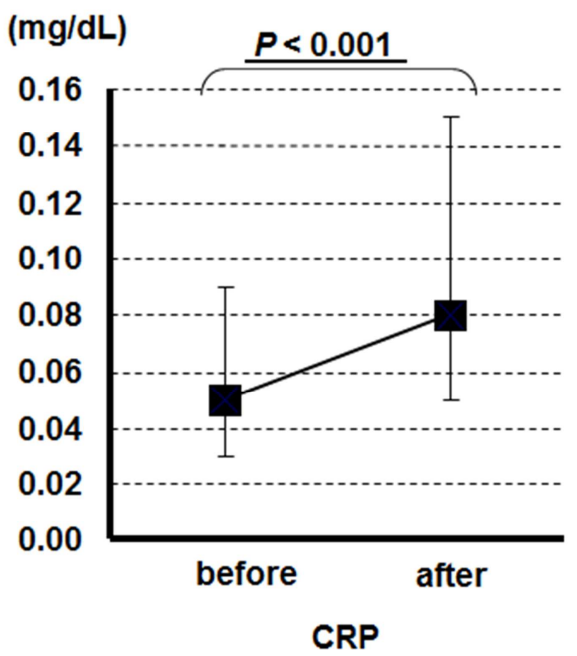

Figure 1. Changes in WBC and CRP before and after colonoscopy in all patients. Median values with quartiles (25\% - 75\%) are presented. P values were determined using the Wilcoxon signed rank test.

In the subgroups, a comparison of results before and after colonoscopy showed that the mean WBC decreased slightly in the P- group (before: $5400 / \mu \mathrm{L}$, after: $4900 / \mu \mathrm{L}, P=0.002$ ) but was not significantly different in the $\mathrm{P}+$ group (before: $5400 / \mu \mathrm{L}$, after: $5400 / \mu \mathrm{L}, P=0.48)$. The difference in $\mathrm{WBC}$ elevation $(\triangle \mathrm{WBC})$ between the $\mathrm{P}-$ and $\mathrm{P}+$ groups was significant $(P=0.004)$ (Figure 2$)$. On the other hand, the CRP level showed a slight elevation in both the $\mathrm{P}$ - and $\mathrm{P}+$ groups

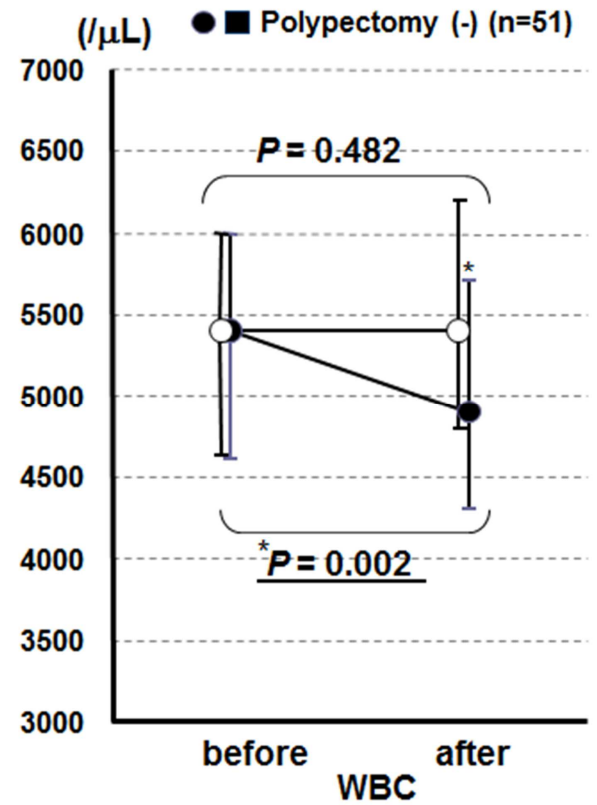

(before: $0.06 \mathrm{mg} / \mathrm{dL}$, after $0.07 \mathrm{mg} / \mathrm{dL}, P=0.003$; before: 0.04 $\mathrm{mg} / \mathrm{dL}$, after: $0.08 \mathrm{mg} / \mathrm{dL}, P<0.001$, respectively). However, the difference in CRP elevation $(\triangle \mathrm{CRP})$ between the $\mathrm{P}$ - and $\mathrm{P}+$ groups was not significant $(P=0.076)$ (Figure 3$)$.

There was a very small correlation between the number of resected polyps in each patient and both $\triangle \mathrm{WBC}(\mathrm{r}=0.307, P=$ $0.001)$ (Figure 4) and $\triangle \mathrm{CRP}(\mathrm{r}=0.245, P=0.010)$ (Figure 5).

$\bigcirc$ Polypectomy (+) (n=58)

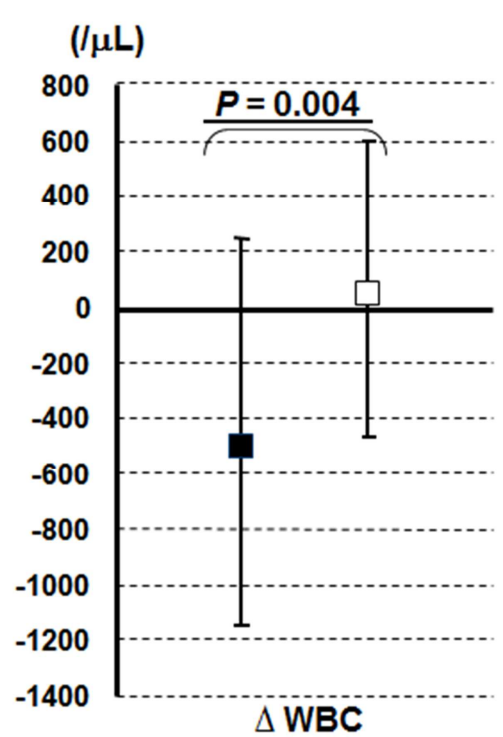

Figure 2. Changes in WBC before and after colonoscopy in the two subgroups. Median values with quartiles (25\% - 75\%) are presented. P values were determined using the Wilcoxon signed rank test or Mann-Whitney test. $\triangle W B C$, white blood cell count elevation. 

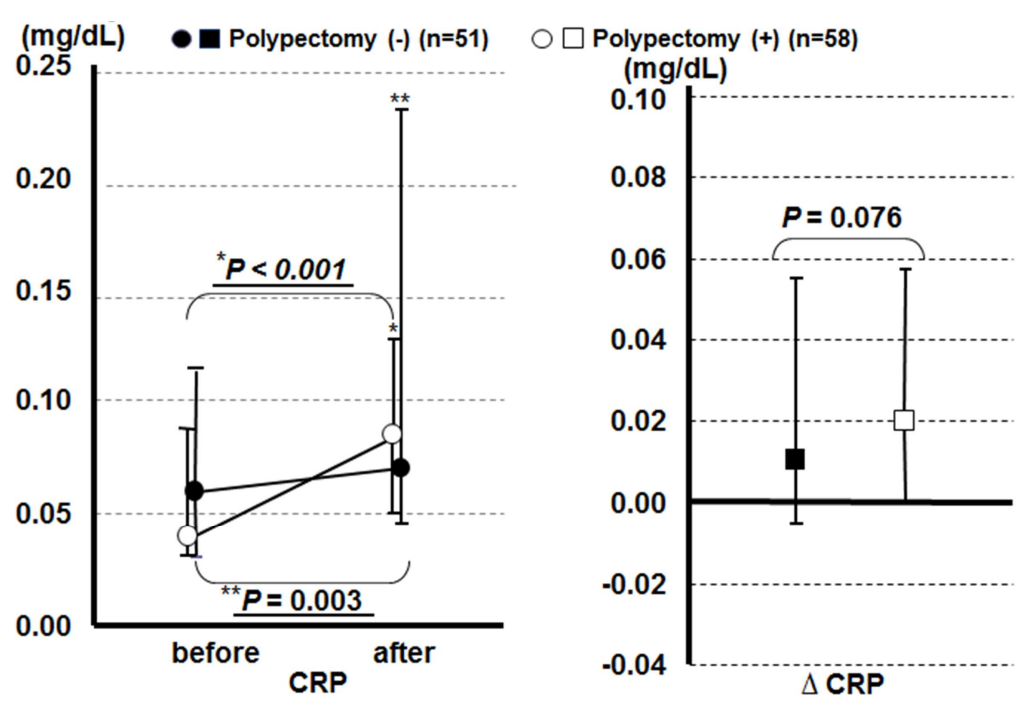

Figure 3. Changes in CRP before and after colonoscopy in the two subgroups. Median values with quartiles (25\% - $75 \%)$ are presented. $P$ values were determined using the Wilcoxon signed rank test or Mann-Whitney test. $\triangle C R P, C$-reactive protein level elevation.

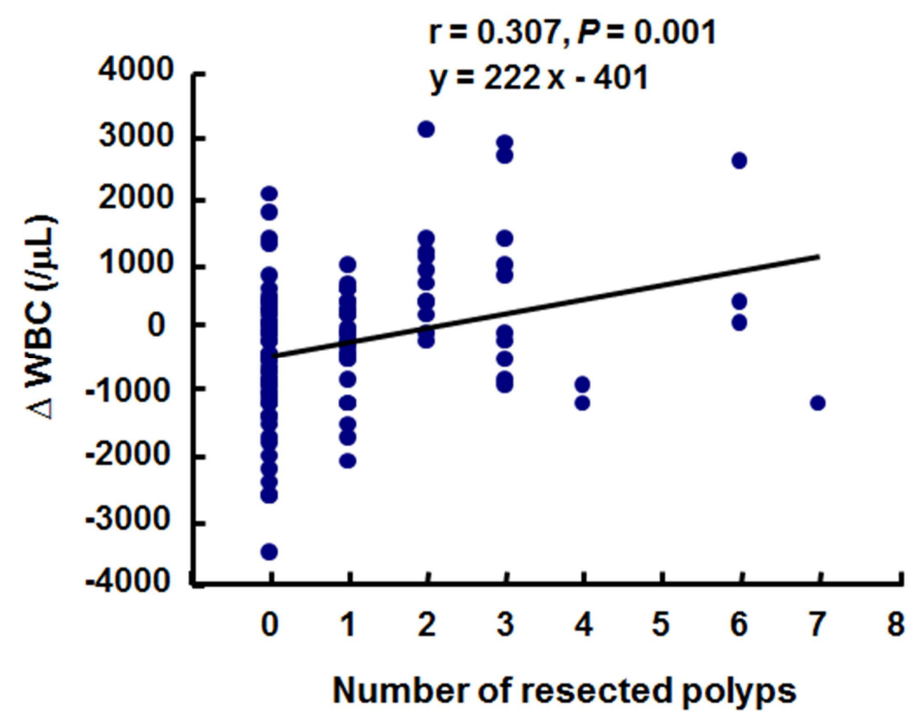

Figure 4. Correlation between the number of resected polyps and $\triangle W B C$ in each patient. Spearman's rank correlation coefficient was used. $\triangle W B C$, white blood cell count elevation.

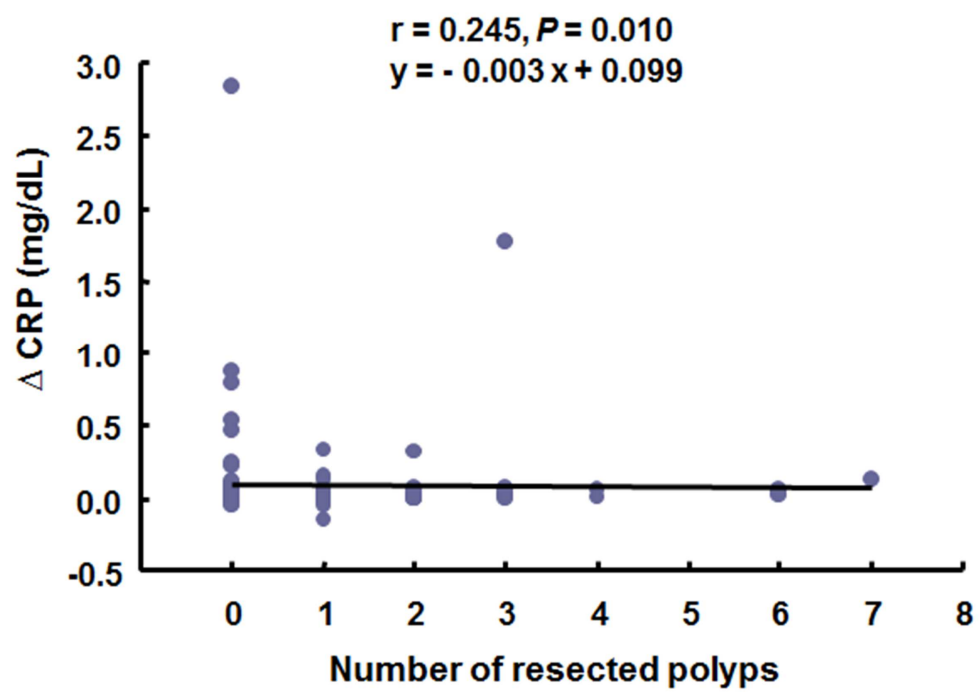

Figure 5. Correlation between the number of resected polyps and $\triangle C R P$ in each patient. Spearman's rank correlation coefficient was used. $\triangle C R P, C$-reactive protein level elevation. 


\section{Discussion}

In this study, physical stress caused by colonoscopy and polypectomy was investigated using two blood-derived damage biomarkers, the WBC and CRP level. The results showed that the WBC was not elevated after colonoscopy even when polypectomy was also performed. In contrast, the CRP level showed elevation both after colonoscopy alone and after polypectomy.

To evaluate physical stress, WBCs and CRP levels in blood were measured, both of which are nonspecific but widely used markers. Changes in the WBC occur rapidly following tissue damage, inflammation, and other abnormal conditions. CRP is one of the acute phase proteins, which are induced within 6-10 hours in response to any tissue-damaging process and/or inflammation [13-15]. It is a liver-derived pentameric protein, and the main stimulus for its secretion is interleukin (IL)-6 derived from macrophages and T cells [15]. The WBC and CRP level were considered to be pertinent for this study because they respond quickly to tissue damage and can be easily measured.

Generally, there are two types of stress, namely psychological and physical stresses. There are several studies on the rapid evaluation of psychological stress in various conditions using biomarkers, such as salivary alpha amylase [16-25] and salivary cortisol [18, 25-27]. However, to our knowledge, only one study has evaluated psychological stress among endoscopy examinees using the biomarker chromogranin A [28]. In comparison, various studies have evaluated physical stress using biomarkers such as salivary alpha amylase [29] and several blood-derived biomarkers, including glucose, cortisol, catecholamine $[30,31]$, somatotropin, insulin, CRP [31], toll-like receptors, IL-6, tumor necrosis factor-alpha, high-sensitivity CRP [32], alpha-defensin [33], IL-8, IL-10, albumin, prealbumin, and total iron-binding capacity [34], but these biomarkers were mostly measured in surgical situations. Only one study has evaluated endoscopic physical stress using blood-derived biomarkers that demonstrated a significant increase in norepinephrine and cortisol concomitant with a significant peak in heart rate and mean arterial blood pressure [30], but it was performed in patients who underwent upper gastrointestinal endoscopy. Yet, no study has evaluated physical stress caused by colonoscopy using blood-derived biomarkers.

Physical stress associated with colonoscopy includes both bowel preparation, which requires consuming as much as two liters of polyethylene glycol solution, and the colonoscopy procedure itself, which seems to cause major stress. Bowel preparation may lead to dehydration or electrolyte imbalance, particularly in elderly patients. Colonoscopy procedure-related physical stress can be caused by air insufflations and by colonoscope manipulation. It is inevitable that during colonoscopy, a large amount of air supplied through the colonoscope extends the large intestine beyond the physiological threshold. In addition, although colonoscopy devices have become more flexible recently, they significantly stretch the intestinal wall and the mesenterium. Carbon dioxide $\left(\mathrm{CO}_{2}\right)$ insufflation can reduce the undesirable symptoms derived from excessive intraluminal air [11, 12], but use of the $\mathrm{CO}_{2}$ insufflation system is not wide spread because of its high cost.

Endoscopic polypectomy, which is considered a type of surgical procedure, is frequently performed during colonoscopy. When polypectomy is performed, a burning effect can cause additional substantial damage to the intestinal wall.

Physical stress from both colonoscopy and endoscopic polypectomy may lead to procedure-related acute minor symptoms after colonoscopy, including abdominal discomfort, gas bloating, nausea, and abdominal pain. Unfortunately, severe complications, such as bleeding and perforation, may also occur, but they are rare. Examiners can ascertain physical stress in patients by their complaints about these symptoms or by changes in their vital signs. However, it is unknown what changes may occur in the blood.

As mentioned above, salivary alpha-amylase is most frequently measured as an immediate stress biomarker because it is a surrogate for sympathetic nerve system activity, but it seems to be more useful when evaluating psychological stress rather than physical stress. In this study, the WBC and CRP level were used as physical stress biomarkers in the blood because they can reach high levels in response to various invasive injuries to the body even if the damage is not severe. Tomizawa et al. reported that an elevated CRP level measured before colonoscopy predicted lower gastrointestinal tract bleeding [35]. This report suggested that an elevated CRP level in response to lower gastrointestinal tract bleeding indicated substantial physical stress.

We assumed that stress from colonoscopy could be strong enough to affect the WBC and CRP level and that polypectomy would add more stress compared to diagnostic colonoscopy alone. Unexpectedly, there was no significant WBC elevation after colonoscopy or polypectomy. On the contrary, the WBC decreased after colonoscopy in the Pgroup, and the cause of which is unclear. However, five patients (including four of the five who underwent polypectomy) showed WBC elevation of $2000 / \mu \mathrm{L}$ or more. This result implies that the WBC could be affected by polypectomy because the burning effect may vary substantially among patients.

As for the CRP level, slight elevations were demonstrated in both the $\mathrm{P}$ - and $\mathrm{P}+$ groups. However, even in the $\mathrm{P}+$ group, no more than three of 58 patients showed CRP elevation after polypectomy beyond the normal upper limit of $0.3 \mathrm{mg} / \mathrm{dL}$. Although both the WBC and CRP level are recognized as rapid biomarkers of damage and inflammation to the body, they may respond differently. It is possible that CRP level is more sensitive to damage or stays abnormal for longer periods than the WBC.

Both WBC and CRP elevation showed only very minor correlations with the number of resected polyps. These results 
may be attributed to the small number of multi-polypectomy cases. Further study should be conducted to clarify the correlation between the number of resected polyps and physical stress.

In conclusion, in elderly individuals, colonoscopy can cause physical stress, which is expressed as elevations in the blood-derived biomarkers. However, these elevations were very minor, as observed in this study, and polypectomy rarely added further physical stress.

Several limitations of this study should be acknowledged: this study was conducted in a single center; the difference of colonoscopy devices used may have affected the results; use of sedation may have masked the physical stress from colonoscopy; total procedure time and burning effect of polypectomy could be different among patients because the skill levels of endoscopy examiners were not the same, although they were all endoscopic specialists; results are only applicable to elderly people aged $\geq 80$ years; and there might be better blood-derived biomarkers than the WBC and CRP level to express physical stress adequately. To evaluate physical stress from different aspects, further studies using other stress markers are required. Furthermore, physical stress from endoscopic submucosal dissection, which is recognized to be a more invasive procedure than polypectomy, should be evaluated as well.

\section{Conclusions}

Physical stress associated with colonoscopy in elderly individuals seemed to be minimal, even if polypectomy was performed, based on blood-derived damage biomarkers such as the WBC and CRP level. On the basis of these results, colonoscopy can be well tolerated and serve as a good examination modality, even for elderly individuals.

\section{Conflict of Interest}

The authors declare that they have no conflicts of interest.

\section{References}

[1] Vital Statistics Japan (Ministry of Health, Labour and Welfare) 2016.

[2] Mandel JS, Bond JH, Church TR et al. Reducing mortality from colorectal cancer by screening for fecal occult blood. New Engl J Med 1993; 328: 1365-1371.

[3] Hardcastle JD, Chamberlain JO, Robinson MHE et al. Randomised controlled trial of fecal-occult-blood screening for colorectal cancer. Lancet 1996; 348: 1472-1477.

[4] Kronborg O, Fenger C, Olsen J, Jorgensen OD, and Sondergaard O. Randomised study of screening for colorectal cancer with faecal-occult-blood test. Lancet 1996; 348: $1467-1471$.

[5] European Colorectal Cancer Screening Guidelines Working Group. European guidelinesfor quality assurance in colorectal cancer screening and diagnosis: Overview and introduction to the full supplement publication. Endoscopy 2013; 45: 51-59.

[6] Shaukat A, Mongin SJ, Geisser MS et al. Long-term mortality after screening for colorectal cancer. New Engl J Med 2013; 369: 1106-1114.

[7] Canadian Task Force on Preventive Health Care. Recommendations on screening for colorectal cancer in primary care. CMAJ 2016; 188: 340-348.

[8] US Preventive Services Task Force. Screening for colorectal cancer. JAMA 2016; 315: 2564-2575.

[9] Nishihara $\mathrm{R}$, Wu $\mathrm{K}$, Lochhead $\mathrm{P}$ et al. Long-term colorectal-cancer incidence and mortality after lower endoscopy. New Engl J Med 2013; 369: 1095-1105.

[10] Whitlock EP, Lin J, Liles E et al. Screening for colorectal cancer: An updated systematic review [Internet]. Agency for Healthcare Research and Quality (US); 2008 Oct. Report No.: 08-05-05124-EF-1.

[11] Iida T, Okamura S, Kakizaki $\mathrm{S}$ et al. Carbon dioxide insufflation reduces the discomfort due to colonoscopy as objectively analyzed by salivary stress markers. Acta Gastroenterol Belg 2013; 76: 219-224.

[12] Kiriyama S, Naitoh H, Fukuchi $M$ et al. Evaluation of abdominal circumference and salivary amylase activities after unsedated colonoscopy using carbon dioxide and air insufflations. J Dig Dis 2015; 16: 747-751.

[13] Kushner I. The phenomenon of the acute phase response. NYAS 1982; 82: 39-48.

[14] Thompson D, Ward AM, and Whicher JT. The value of acute phase protein measurements in clinical practice. Ann Clin Biochem 1992; 29: 123-131.

[15] Clos TWD. Function of C-reactive protein. Ann Med 2000; 32: 274-278.

[16] Gilman SC, Fischer GJ, Biersner RJ, Thornton RD, and Miller DA. Human parotid gland alpha-amylase secretion as a function of chronic hyperbaric exposure. Undersea Biomed Res 1979; 6: 303-307.

[17] Chatterton RT Jr, Vogelsong KM, Lu Y, Ellman AB, and Hudgens GA. Salivay $\alpha$-amylase as a measure of endogenous adrenergic activity. Clin Physiol 1996; 16: 433-448.

[18] Takai N, Yamaguchi M, Aragaki T, Eto K, Uchihashi K et al. Effect of psychological stress on the salivary cortisol and amylase levels in healthy young adults. Arch Oral Biol 2004; 49: 963-968.

[19] Rohleder N, Nater UM, Wolf JM, Ehlert U, and Kirschbaum C. Psychosocial stress-induced activation of salivary alpha-amylase. An indicator of sympathetic activity? Ann NY Acad sci 2004; 1032: 258-263.

[20] Yamaguchi M, Deguchi M, Wakasugi J et al. Hand-held monitor of sympathetic nervous system using salivary amylase activity and its validation by driver fatigue assessment. Biosens Bioelectron 2006; 21: 1007-1014.

[21] Stegeren AV, Rohleder N, Everaerd W, and Wolf OT. Salivary alpha amylase as marker for adrenergic activity during stress: Effect of betablckade. Psychoneuroendocrinology 2006; 31: 137-141. 
[22] Noto Y, Sato T, Kudo M, Kurata K, and Hirota K. The relationship between salivary biomarkers and state-trait anxiety inventory score under mental arithmetic stress: A pilot study. Anesth Analg 2005; 101: 1873-1876.

[23] Nater UM, Marca RL, Florin L et al. Stress-induced changes in human salivary alpha-amylase activity-associations with adrenergic activity. Psychoneuroendocrinology 2006; 31: 49-58.

[24] Robles TF, Shetty V, Zigler CM et al. The feasibility of ambulatory biosensor measurement of salivary alpha amylase: Relationships with self-reported and naturalistic psychological stress. Biol Psychol 2011; 86: 50-56.

[25] Engert V, Vogel S, Efanov SI et al. Investigation into the crosscorrelation of salivary cortisol and alpha-amylase responses to psychological stress. Psychoneuroendocrinology 2011; 36: 1294-1302.

[26] Kirschbaum C, and Hellhammer DH. Salivary cortisol in psychobiological research: An overview. Neuropsychobiology 1989; 22: 150-169.

[27] Eck MV, Berkhof H, Nicolson N, and Sulon J. The effects of perceived stress, traits, mood states, and stressful dialy events on salivary cortisol. Psychosom Med 1996; 58: 447-458.

[28] Fujimoto S, Nomura M, Niki M et al. Evaluation of stress reactions during upper gastrointestinal endoscopy in elderly patients: assessment of mental stress using chromogranin A. J Med Invest 2007; 54: 140-4.
[29] Robles TF, Sharma R, Park KS, Harrell L, and Yamaguchi M. Utility of salivary biosensor for objective assessment of surgery-related stress. J Oral Maxillofac Surg 2012; 70: 2256-2263.

[30] Tonnesen H, Puggaard L, Braagaard J, Ovesen H, and Rasmussen V. Stress Response to endoscopy. Scand J Gastroenterol 1999; 6: 629-631.

[31] Luo K, Li JS, Li LT, Wang KH, and Shun JM. Operative stress response and energy metabolism after laparoscopic cholecystectomy compared to open surgery. World J Gastroenterol 2003; 9: 847-850.

[32] Tsimogiannis KE, Tellis CC, Tselepis AD, Pappas-Gogos GK, and Tsimoyiannis EC. Toll-like receptors in the inflammatory response during open and laparoscopic colectomy for colorectal cancer. Surg Endosc 2012; 26: 330-336.

[33] Tsimogiannis KE, Telis K, Tselepis A, Pappas-Gogos GK, and Tsimoyiannis EC. $\alpha$-Defensin Expression of Inflammatory Response in Open and Laparoscopic Colectomy for Colorectal Cancer. World J surg 2011; 35: 1911-1917.

[34] Andersson B, Ansari D, Norden M, Nilsson J, and Andersson R. Surgical stress response after colorectal resection. Int surg 2013; 98: 292-299.

[35] Tomizawa M, Shinozaki F, Hasegawa R et al. Elevated C-reactive protein level predicts lower gastrointestinal tract bleeding. Biomed Rep 2016; 4: 711-714. 